https://helda.helsinki.fi

\title{
The presence of minor salivary glands in the peritonsillar space
}

\section{Kaltiainen, Enni}

2017-11

Kaltiainen , E , Wiksten , J , Aaltonen , L-M , Ilmarinen , T , Hagstrom , J \& Blomgren , K 2017 , ' The presence of minor salivary glands in the peritonsillar space ' , European Archives of Oto-Rhino-Laryngology , vol. 274 , no. 11 , pp. 3997-4001 . https://doi.org/10.1007/s00405-017-4738-x

http://hdl.handle.net/10138/298076

https://doi.org/10.1007/s00405-017-4738-x

publishedVersion

Downloaded from Helda, University of Helsinki institutional repository.

This is an electronic reprint of the original article.

This reprint may differ from the original in pagination and typographic detail.

Please cite the original version. 


\title{
The presence of minor salivary glands in the peritonsillar space
}

\author{
Enni Kaltiainen ${ }^{1}$ (1) $\cdot$ Johanna Wikstén ${ }^{1} \cdot$ Leena-Maija Aaltonen $^{1} \cdot$ Taru Ilmarinen $^{1}$ • \\ Jaana Hagström ${ }^{2} \cdot$ Karin Blomgren $^{1}$
}

Received: 12 April 2017 / Accepted: 5 September 2017 / Published online: 12 September 2017

(C) Springer-Verlag GmbH Germany 2017

\begin{abstract}
Peritonsillar abscess (PTA) is traditionally considered only a purulent complication of acute tonsillitis (AT), but may be related to infection of minor salivary glands. We analysed the presence of peritonsillar minor salivary glands and inflammation patterns in 114 adult tonsils representing three patient groups: recurrent AT, chronic tonsillitis (CT), and PTA. Samples acquired from elective tonsillectomies were stored in formalin, and after preparation were microscopically examined for inflammation and fibrotic changes. Clinical features and histological characteristics were compared between the groups. Of all tonsils, the minor salivary glands were present in 77 (67.5\%). Glands located near the tonsillar tissue showed signs of infection in $73(94.8 \%)$, while only $3(15.0 \%)$ of 20 glands located deeper in the peritonsillar space were infected. Compared to patients with recurrent AT and CT, those with PTA more often presented with periductal inflammation, $p<0.011$ (PTA $82.1 \%$, AT $42.9 \%$, and CT $63.6 \%$ ). The majority of our 114 tonsillectomy specimens, collected from patients with AT, CT, or PTA, presented with infected minor salivary glands, and inflammation of the peritonsillar space glands was evident. To further elucidate the association between these glands and PTA, tonsillar samples should be collected and analysed from patients during the acute phase of infection.
\end{abstract}

Enni Kaltiainen

enni.kaltiainen@gmail.com

1 Department of Otorhinolaryngology-Head and Neck Surgery, Helsinki University Hospital, Helsinki, Finland

2 Department of Pathology, Helsinki University Hospital, Helsinki, Finland
Keywords Peritonsillar abscess - Weber's glands · Acute tonsillitis · Chronic tonsillitis · Tonsillectomy · Minor salivary glands

$\begin{array}{ll}\text { Abbreviations } \\ \text { PTA } & \text { Peritonsillar abscess } \\ \text { ORL } & \text { Otorhinolaryngological } \\ \text { AT } & \text { Acute tonsillitis } \\ \text { TE } & \text { Tonsillectomy } \\ \text { CT } & \text { Chronic tonsillitis }\end{array}$

\section{Introduction}

Peritonsillar abscess (PTA) is the most common otorhinolaryngological (ORL) infection requiring hospitalization $[1,2]$. Despite the high incidence, the aetiology of PTA is controversial. PTA is traditionally regarded as a purulent complication of acute tonsillitis (AT). However, no evidence supports this theory [3]. A presumption was raised over two decades ago that the development of PTA could be associated with an infection of Weber's glands [1,2].

Weber's glands (group of 20-25 mucous salivary glands) are located in the peritonsillar space [2]. The precise location of these minor salivary glands and the histological findings in the tonsil of PTA patients are presented only in few, uncontrolled studies [2-4].

Minor salivary glands are localized at the upper, middle, and lower portions of the peritonsillar space [3]. Patients with PTA present with inflammation and fibrosis of minor salivary glands in the inflamed tissue surrounding the tonsils $[4,5]$. El Saied et al. showed that both serum and pus amylase levels are highly elevated in patients with PTA, compared with deep neck abscesses and dental abscesses $[6,7]$. 
These observations strengthen the theory about the role of infection of minor salivary glands in PTA.

Recently, in a large cohort, PTA incidence did not increase during winter when AT infections occurred more often [8]. It is also known that AT is usually bilateral, whereas PTA is, with very few exceptions, unilateral $[9$, 10]. PTA can also occur in patients with no tonsil tissue left after tonsillectomy (TE) [11]. All these results support the theory that PTA is not necessarily a complication of AT, and therefore should not be treated by TE. Conversely, it may originate from the minor salivary glands, and factors such as smoking or periodontal disease, or other currently unidentified causes, may be the predisposing factors [12, 13].

The purpose of this study was to explore the possible differences in tonsil tissue between patients with PTA, AT, and chronic tonsillitis (CT). Inflammation and erosion of the minor salivary glands in PTA patients would support the role of minor salivary glands in the pathogenesis of PTA.

\section{Materials and methods}

The study material consisted of tonsil samples of 278 patients, who had undergone TE at the ORL clinic of Helsinki University Hospital in 2012 and 2015. Indications for TE were recurrent AT, CT, and PTA. The tonsils were originally collected for a human papilloma virus study. The Ethics Committee of Helsinki University Hospital approved the former and present study. All patients had given their informed written consent when participating in the original study for the use of their samples also in future studies.

For the current study, a total of 124 samples were selected from the original 278 patients and divided into three groups based on the diagnosis: PTA, CT, and AT. First, we selected all PTA patients, fulfilling the inclusion criteria, from the original 278 patients. For the two control groups, we included all AT and CT patients with the same age range as PTA patients (18.47-69.48). Hence, we studied three groups with nearly the same size and the same age range. Exclusion criteria were age under 18 years, previous tonsillotomy, insufficient amount of tonsil tissue for histological analysis, a suspicion of malignancy, or multiple ORL diagnosis. Patients' medical records were reviewed. All patients had completed a questionnaire including questions concerning their general overall health, medications, and smoking habits. Patients typically underwent TE approximately 6 months (mean 6.25 months, median 6 months, min 1 month, max 13 months) after the diagnosis and decision to have the operation. During TE, one tonsil was resected as a specimen for this study. Immediately after TE, the tonsils were formalin fixed and after fixation cut into two to four sections depending on the tonsil's size. The tonsils were embedded in paraffin. For histological examination, $4 \mu \mathrm{m}$ slides were cut from paraffin-embedded tissue blocks and stained with haematoxylin and eosin for histological examination. A total of 114 tonsils were available for analyses: we analysed two to four slides per patient depending on the tonsil size. All prepared slides were examined in the study for the presence of minor salivary glands as well as the location of the glands. Inflammation, degenerative and fibrotic changes of minor salivary glands, as well as the possible histological changes in the salivary ductus, were also reported. Inflammation was defined as leukocyte infiltration into minor salivary glands. The degree of inflammation was graded as none, moderate, or severe. Both periductal and acinar inflammations were separately evaluated. Statistical calculations were performed by NCSS 8 (Kaysville, Utah, USA) 2012 statistical analysis software using the Chi square-test. $p$ values $<0.05$ were considered to be significant.

\section{Results}

Altogether, 114 patients were included in this study. Age, gender, data on smoking habits (currently, never, previously), duration of the symptoms prior to PTA, and prior antibiotic treatment were obtained from the patient records (Table 1). Alcohol consumption was not considered in the questionnaire. There was no mention about alcohol overuse or periodontal disease in any patient record reviewed.

Minor salivary glands were identified in 77 (67.5\%) tonsil samples. In 20 (17.5\%), minor salivary glands were observed also deeper in the smooth muscle tissue. There was no significant difference in the presence of minor salivary glands between the three subgroups $(p=0.372)$. Glands located superficially, near the tonsillar tissue, showed signs of infection in $73(94.8 \%)$ patients. Only three $(15.0 \%)$ of the deeper glands in the peritonsillar space were inflamed. The inflammation signs were stronger near the tonsillar tissue than in the deeper peritonsillar space. The PTA group demonstrated more intense periductal inflammation $(82.1 \%)$ than the AT $(57.1 \%)$ or CT group (42.9\%) $(p=0.011)$ (Fig. 1). The amount of inflammation around the acinar structures was equal in all subgroups, PTA (50\%, AT (60\%) and CT $(57.1 \%)(p=0.725)$. There were no statistical differences in the presence of minor salivary glands $(p=0.970)$ or in periductal inflammation signs $(p=0.211)$ between patients who had undergone TE after the first PTA episode and patients who had suffered from multiple (2 or 3) PTAs before TE. Altogether 21 (53.8\%) of the PTA patients analysed tonsils were from the same side where the abscess had existed. They demonstrated salivary glands in $81.0 \%$ of the samples, while analysing tonsils from the non-abscess side we observed minor salivary glands in only $61.1 \%$ of the samples $(p=0.170)$. Inflammation of the salivary glands was shown in $94.1 \%$ of the salivary glands analysed from the same side 
Table 1 The characteristics of 114 patients collected from patient records
Fig. 1 Periductal inflammation of the minor salivary gland on the right. Leukocyte infiltration around the ductus marked with arrows. Non-inflamed minor salivary gland on the left side of the picture as a control. Non-inflamed ductus and noninflamed minor salivary gland marked with arrows

\begin{tabular}{|c|c|c|c|}
\hline Characteristics & PTA group $(n=39)$ & AT group $(n=50)$ & CT group $(n=25)$ \\
\hline \multicolumn{4}{|l|}{ Age, years } \\
\hline Median & 23,97 & 26,03 & 32,19 \\
\hline Range & $18,49-69,48$ & $18,67-50,05$ & $19,70-49,32$ \\
\hline Male & 16 & 35 & 5 \\
\hline Female & 23 & 15 & 20 \\
\hline Smoker & 16 & 20 & 7 \\
\hline Ex-smoker & 12 & 5 & 3 \\
\hline \multicolumn{4}{|c|}{ Tonsillitis prior to TE } \\
\hline None & 18 & 0 & \\
\hline $1-3$ & 7 & 3 & \\
\hline$>3$ & 8 & 47 & \\
\hline Missing & 6 & 0 & \\
\hline \multicolumn{4}{|l|}{ Prior antibiotics } \\
\hline 1. PTA & 16 & & \\
\hline Missing & 4 & & \\
\hline 2. PTA & 3 & & \\
\hline Missing & 22 & & \\
\hline \multicolumn{4}{|c|}{ Duration (days) of symptoms (throat pain, fever, hoarseness) prior to PTA } \\
\hline \multicolumn{4}{|l|}{ 1. PTA } \\
\hline $1-6$ & 14 & & \\
\hline$>6$ & 18 & & \\
\hline Missing & 7 & & \\
\hline \multicolumn{4}{|l|}{ 2. PTA } \\
\hline $1-6$ & 18 & & \\
\hline$>3$ & 6 & & \\
\hline Missing & 15 & & \\
\hline
\end{tabular}

PTA peritonsillar abscess, $A T$ acute tonsillitis, $C T$ chronic tonsillitis, $T E$ tonsillectomy, 1. PTA patient's first peritonsillar abscess episode, 2. PTA patient's second peritonsillar abscess episode, missing no mention in patient charts
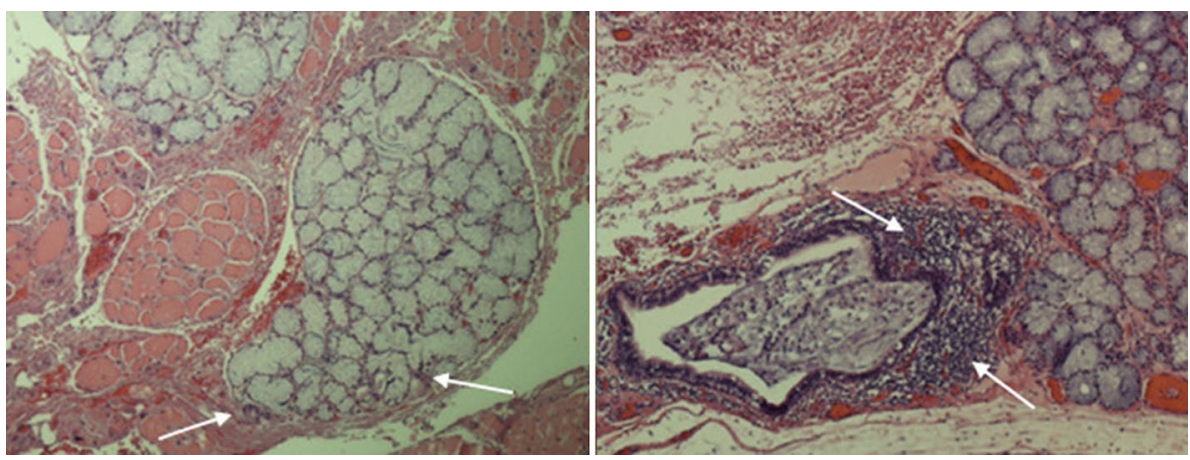

than the existence of abscess and in 100\% of those analysed from the non-infected side. The difference was not statistically significant $(p=0.413)$. Fibrosis and degeneration were observed only in a couple of specimens (fibrosis in 20 , degeneration in 8), so subgroup analysis was unreliable. Actinomyces-like bacteria existed in $62(54.5 \%)$ specimens (Fig. 2). The presence of actinomyces-like bacteria was the same in all subgroups.
In our data, smoking habits had no effect on the presence of salivary glands (superficial $67.5 \%$ or deeper $18.6 \%$ ) ( $p=0.964, p=0.171)$, inflammation of salivary glands (periductal or acinar) $(p=0.758, p=0.115)$, or the presence of actinomyces $(53.5 \%, p=0.988)$. The effects of smoking on the duration of symptoms before PTA diagnosis could not be analysed reliably because of the small number of evaluated patients. 


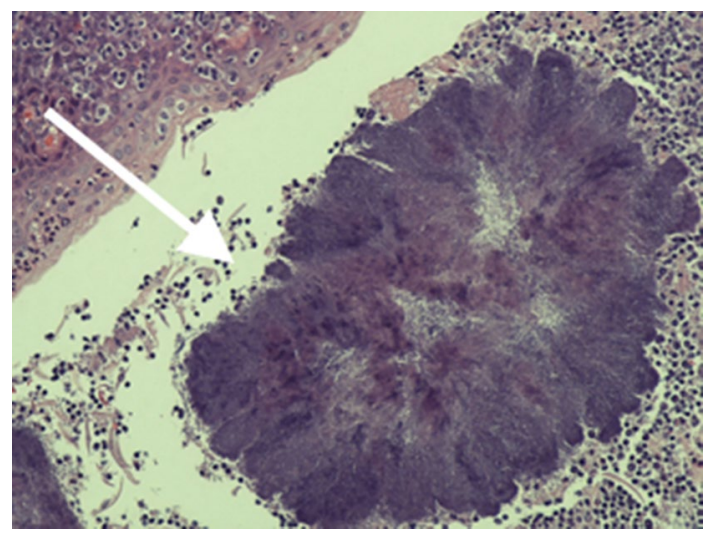

Fig. 2 Actinomyces-like bacteria in the tonsil tissue, marked with arrow

\section{Discussion}

We identified minor salivary glands in most $(67.5 \%)$ of the 114 tonsil specimens collected after TE due to recurrent AT, CT, or PTA. In addition to superficially located, near the tonsil, salivary glands, we also detected minor salivary glands deeper in the tissue between the muscle fibres, which has not been reported in previous studies. We found a significant difference in periductal infection between the PTA group and AT or CT groups.

In this study, we have proven the speculation that minor salivary glands are located close to the tonsils with a commendable number of samples. The fact that the tonsils were collected after a mean 6 months' waiting period, especially concerning the PTA samples, may be considered to be a limitation of the study. We were unable to evaluate the glands' location accurately as in the lower, upper, or middle portions of the peritonsillar space, because specimens were not marked during TE. We, however, identified minor salivary glands in multiple locations in the peritonsillar space which supports Kraitrakul et al.'s findings on minor salivary glands being located all over the peritonsillar space [3].

Kraitrakul et al. have previously identified small salivary glands in $96.4 \%$ of all patients, but they analysed both tonsils while we analysed only one per patient. Observing only one tonsil, they found small salivary glands from 87.3 to $90.9 \%$ of patients which is quite concordant with our findings. Also, the fact that a variety of surgeons performed the TEs may impact the number of glands found depending on how the tonsil was dissected. In Kraitrakul et al.'s study, minor salivary glands were found from all portions of the peritonsillar space. Though the distribution varied between the patients, there was no predominant pattern [3]. In our study, inflammation of the glands in the peritonsillar tissue was evident, and the PTA group demonstrated more periductal inflammation than other subgroups. Few small, uncontrolled studies have shown an undefined inflammation of minor salivary glands among PTA patients [2, 4]. Kraitrakul et al. also reported a slight inflammation in the periductal space, but failed to observe these changes in their three PTA patients, or any fibrotic or degenerative changes [3]. In our study, we also identified some fibrotic and degenerative changes, but their significance remains unclear due to small sample size. In recent years, the aetiology of PTA has been speculated but no consensus exists. Findings supporting the different aetiologies have been introduced: High amylase levels in the peritonsillar pus aspirate and the presence of minor salivary glands with signs of infection in the peritonsillar space indicate the role of minor salivary glands in PTA. The fact that the same pathogens are found both in the pus aspirate and in both tonsils supports the aetiology of bilateral tonsillar infection behind PTA. The tonsillar aetiology is also supported by the possible preventive effect of antibiotic treatment in the development of PTA [1, 3, 9, 14-16].

The PTA group's age distribution in our study was similar to that reported in the literature. The distribution of gender in our study differs from previous studies which have demonstrated male predominance [17-19]. The age of the other subgroups (AT, CT) could not be analysed reliably, because they were formed based on the age range. Previous studies have, however, reported equal range and median age in AT, as in our study [20]. Several studies have showed the predominance of smoking among PTA patients compared to the whole population and our results agree with these. We also noted a predominance of ex-smokers (quit $<15$ years ago) in PTA patients [12, 21, 22].

In PTA and deep neck abscess patients, the prevalence of actinomyces-like bacteria has been shown to be $23 \%$ when measured/analysed from pus [23]. Histological analysis of tonsillar tissue in CT and sleep apnoea patients has revealed the presence of actinomyces-like bacteria in 35.6-40.6\%. These numbers are a little lower than those in our data, but are concordant with the previous literature [24, 25]. A recent study also surprisingly showed that the proportion of actinomyces-like bacteria of all bacteria was lower in smokers [26]. In our material, we were unable to find a single factor affecting the presence of actinomyces-like bacteria in peritonsillar tissue. As such, the role of actinomyces-like bacteria in tonsillar diseases remains unclear.

\section{Conclusion}

When observing tonsil specimens collected after TE due to recurrent AT, CT, or PTA, minor salivary glands could be identified in most $(67.5 \%)$ of the 114 samples. The infected glands were located mostly superficially, but deeper location of the glands could also be seen. Periductal inflammation in the small salivary glands was prominent in the PTA group 
when compared with the AT and CT groups. These findings support the theory that PTAs may indeed originate from the infection of minor salivary glands. To better understand the clinical impact of these glands and any difference between patient groups, tonsillar samples from patients with acute tonsillar or peritonsillar infection should be collected and analysed.

\section{Compliance with ethical standards}

Funding This study was funded by the Helsinki University Hospital, Department of Otorhinolaryngology-Head and Neck Surgery and University of Helsinki, Finland (Grant number 1149009), and Biomedicum Helsinki Foundation, Finland (no Grant number).

Conflict of interest None of the authors has any financial or other relationship that might lead to conflict of interests.

Ethical approval The research involves human participants and all procedures performed in the study involving human participants were in accordance with the ethical standards of the institutional and/ or national research committee (the Ethics Committee of Helsinki University Hospital) and with the 1964 Helsinki Declaration and its later amendments or comparable ethical standards. The tonsils were originally collected for a human papilloma virus study. The Ethics Committee of Helsinki University Hospital approved the former and present study.

Informed consent All patients had given their informed written consent when participating in the original study for the use of their samples also in future studies.

\section{References}

1. Powell EL et al (2013) A review of the pathogenesis of adult peritonsillar abscess: time for a re-evaluation. J Antimicrob Chemother 68(9):1941-1950

2. Passy V (1994) Pathogenesis of peritonsillar abscess. Laryngoscope 104(2):185-190

3. Kraitrakul S, Sirithunyaporn S, Yimtae K (2001) Distribution of minor salivary glands in the peritonsillar space. J Med Assoc Thail 84(3):371-378

4. Chen Z, Zhou C, Chen J (1997) Investigation of the infectious route of peritonsillar abscess. Zhonghua Er Bi Yan Hou Ke Za Zhi 32(4):245-246

5. Blair AB, Booth R, Baugh R (2015) A unifying theory of tonsillitis, intratonsillar abscess and peritonsillar abscess. Am J Otolaryngol 36(4):517-520

6. El-Saied S et al (2014) A comparison between amylase levels from peritonsillar, dental and neck abscesses. Clin Otolaryngol 39(6):359-361
7. El-Saied S et al (2012) Involvement of minor salivary glands in the pathogenesis of peritonsillar abscess. Otolaryngol Head Neck Surg 147(3):472-474

8. Kordeluk $\mathrm{S}$ et al (2011) Relation between peritonsillar infection and acute tonsillitis: myth or reality? Otolaryngol Head Neck Surg 145(6):940-945

9. Klug TE et al (2016) Peritonsillar abscess: complication of acute tonsillitis or Weber's glands infection? Otolaryngol Head Neck Surg 155(2): 199-207

10. Mazur E et al (2015) Epidemiology, clinical history and microbiology of peritonsillar abscess. Eur J Clin Microbiol Infect Dis 34(3):549-554

11. Farmer SE, Khatwa MA, Zeitoun HM (2011) Peritonsillar abscess after tonsillectomy: a review of the literature. Ann R Coll Surg Engl 93(5):353-355

12. Klug TE et al (2013) Smoking promotes peritonsillar abscess. Eur Arch Otorhinolaryngol 270(12):3163-3167

13. Georgalas $\mathrm{C}$ et al (2002) The association between periodontal disease and peritonsillar infection: a prospective study. Otolaryngol Head Neck Surg 126(1):91-94

14. Dunn N et al (2007) Use of antibiotics for sore throat and incidence of quinsy. Br J Gen Pract 57(534):45-49

15. Del Mar CB, Glasziou PP, Spinks AB (2006) Antibiotics for sore throat. Cochrane Database Syst Rev (4):CD000023

16. Klug TE et al (2011) Significant pathogens in peritonsillar abscesses. Eur J Clin Microbiol Infect Dis 30(5):619-627

17. Bovo R, Barillari MR, Martini A (2016) Hospital discharge survey on 4,199 peritonsillar abscesses in the Veneto region: what is the risk of recurrence and complications without tonsillectomy? Eur Arch Otorhinolaryngol 273(1):225-230

18. Sunnergren O, Swanberg J, Molstad S (2008) Incidence, microbiology and clinical history of peritonsillar abscesses. Scand J Infect Dis 40(9):752-755

19. Hanna BC et al (2006) The epidemiology of peritonsillar abscess disease in Northern Ireland. J Infect 52(4):247-253

20. Gahleitner $\mathrm{C}$ et al (2016) Predisposing factors and management of complications in acute tonsillitis. Acta Otolaryngol 136(9):964-968

21. Marom T et al (2010) Changing trends of peritonsillar abscess. Am J Otolaryngol 31(3):162-167

22. Hidaka $\mathrm{H}$ et al (2011) Precipitating factors in the pathogenesis of peritonsillar abscess and bacteriological significance of the Streptococcus milleri group. Eur J Clin Microbiol Infect Dis 30(4):527-532

23. Brook I (2004) Microbiology and management of peritonsillar, retropharyngeal, and parapharyngeal abscesses. J Oral Maxillofac Surg 62(12): 1545-1550

24. Ashraf MJ et al (2011) Relation between actinomycosis and histopathological and clinical features of the palatine tonsils: an iranian experience. Iran Red Crescent Med J 13(7):499-502

25. Toh ST, Yuen HW, Goh YH (2007) Actinomycetes colonization of tonsils: a comparative study between patients with and without recurrent tonsillitis. J Laryngol Otol 121(8):775-778

26. Peruzzo DC et al (2016) Impact of smoking on experimental gingivitis. A clinical, microbiological and immunological prospective study. J Periodontal Res 51(6):800-811 